\title{
All-optical control of molecular fluorescence
}

\author{
David S. Bradshaw and David L. Andrews* \\ Nanostructures and Photomolecular Systems, School of Chemical Sciences, University of East Anglia, Norwich NR4 7TJ, United Kingdom
}

(Received 12 May 2009; revised manuscript received 6 November 2009; published 29 January 2010)

\begin{abstract}
We present a quantum electrodynamical procedure to demonstrate the all-optical control of molecular fluorescence. The effect is achieved on passage of an off-resonant laser beam through an optically activated system; the presence of a surface is not required. Following the derivation and analysis of the all-optical control mechanism, calculations are given to quantify the significant modification of spontaneous fluorescent emission with input laser irradiance. Specific results are given for molecules whose electronic spectra are dominated by transitions between three electronic levels, and suitable laser experimental methods are proposed. It is also shown that the phenomenon is sensitive to the handedness of circularly polarized throughput, producing a conferred form of optical activity.
\end{abstract}

DOI: 10.1103/PhysRevA.81.013424

PACS number(s): 33.50.-j, 31.30.jn, 42.50.Ct, 42.65.An

\section{INTRODUCTION}

The ability to control the fluorescent decay of molecules is a subject of much present interest. Recent studies of mechanisms that significantly modify the rates of decay have, for example, focused on applications of a strong static field through subwavelength apertures [1], or the engagement of surface electric fields and plasmons [2-6]. Fluorescence emission spectra have also been shown to vary under the effect of a local electromagnetic field within a metal microcavity [7]. Under normal circumstances, fluorescence occurs through spontaneous emission, and each individual process involves a single matter-photon interaction (as represented by the energy diagram of Fig. 1); as such, theory can be cast in terms of first-order time-dependent perturbation theory [8]. When excited molecules are subject to the throughput of suitably resonant laser light, the emission acquires a stimulated character but it remains a first-order interaction; in the language of quantum electrodynamics (QED), its probability is weighted by the occupation number of the input radiation mode. The phenomenon has found analytical applications in stimulated emission depletion spectroscopy $[9,10]$.

It now emerges that the intensity of fluorescence is strongly modified by a completely off-resonant probe laser beam of sufficient intensity, through a nonlinear optical coupling mechanism. Here, there is no net absorption or stimulated emission, but elastic forward scattering-photons are annihilated and created into the same radiation mode, which thus emerges unchanged. Such events can engage by nonlinear coupling with the fluorescence emission, a mechanism that entails three simultaneous matter-photon interactions (Fig. 2); we emphasize that the interaction of the off-resonant beam with the molecule is concurrent with the fluorescence emission, in other words the described mechanism is not stepwise. It is noteworthy that this process is significantly simpler in its experimental demands than all-optical switching mechanisms work on resonance energy transfer [11-13].

In this article, the nature of the nonlinear coupling is fully described and its characteristics, including a manifestation of chirality, are analyzed. In the following section, the funda-

*david.andrews@physics.org mental quantum electrodynamical formulation is presented. Sec. III addresses the general theory of the all-optical control mechanism, constructed in a quantum amplitude form, which includes a derivation of the relevant expressions. Sec. IV develops more specific results for application to three-level systems, and order-of-magnitude calculations are performed to quantify the effect; the analysis concludes in Sec. V with identification of a new form of conferred circular dichroism, manifest in molecules with suitably disposed transition dipole moments.

\section{QED FORMULATION}

The following analysis is based on standard textbook methods of QED [14], employing the Power-Zienau-Woolley formulation [15]. To begin, the nonrelativistic Hamiltonian of a system comprising molecules labeled $\xi$ is defined, in multipolar form, as follows:

$$
H=\sum_{\xi} H_{\mathrm{mol}}(\xi)+\sum_{\xi} H_{\mathrm{int}}(\xi)+H_{\mathrm{rad}},
$$

where $H_{\text {mol }}$ is the molecular Hamiltonian, $H_{\text {rad }}$ is the radiation Hamiltonian, and $H_{\text {int }}$ is the Hamiltonian representing the interaction of the radiation field with molecule $\xi$. The eigenstates, $|n\rangle$, of a basis Hamiltonian given by Eq. (1) excluding $H_{\text {int }}$, form a composite set expressible in the form

$$
|n\rangle=\left|\operatorname{mol}_{n}\right\rangle\left|\operatorname{rad}_{n}\right\rangle \equiv\left|\operatorname{mol}_{n} ; \operatorname{rad}_{n}\right\rangle .
$$

Here, $\left|\operatorname{mol}_{n}\right\rangle$ defines the status of all molecules comprising a product of state vectors for each molecule $\xi$, and $\left|\operatorname{rad}_{n}\right\rangle$ is the radiation (number) state. In the electric-dipole approximation, $H_{\text {int }}(\xi)$ is given by

$$
H_{\text {int }}(\xi)=-\sum_{\xi} \boldsymbol{\mu}(\xi) \cdot \mathbf{e}^{\perp}\left(\mathbf{R}_{\xi}\right),
$$

where the electric-dipole moment operator, $\boldsymbol{\mu}(\xi)$, operates on molecular states, $\left|\operatorname{mol}_{n}\right\rangle$, and the transverse electric field operator, $\mathbf{e}^{\perp}\left(\mathbf{R}_{\xi}\right)$, operates on $\left|\operatorname{rad}_{n}\right\rangle$. The latter involves a summation over all wave vectors, $\mathbf{p}$, and polarizations, $\lambda$, and is usually written as the following mode expansion:

$$
\begin{aligned}
\mathbf{e}^{\perp}\left(\mathbf{R}_{\xi}\right)= & \mathrm{i} \sum_{\mathbf{k}, \lambda}\left(\frac{\hbar c k}{2 \varepsilon_{0} V}\right)^{1 / 2}\left[\mathbf{e}^{(\lambda)}(\mathbf{k}) a^{(\lambda)}(\mathbf{k}) e^{\mathrm{i}\left(\mathbf{k} \cdot \mathbf{R}_{\xi}\right)}\right. \\
& \left.-\overline{\mathbf{e}}^{(\lambda)}(\mathbf{k}) a^{\dagger(\lambda)}(\mathbf{k}) e^{-\mathrm{i}\left(\mathbf{k} \cdot \mathbf{R}_{\xi}\right)}\right]
\end{aligned}
$$




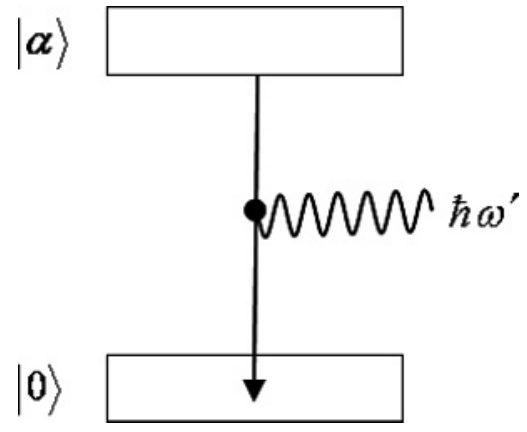

FIG. 1. Energy-level representation for spontaneous fluorescence. Electronic states (and their vibrational manifolds) are signified by the boxes, the wavy line is the emitted fluorescence $\left(\hbar \omega^{\prime}\right)$, and the black vertical arrow is a transition due to the emission. States $|0\rangle$ and $|\alpha\rangle$ denote the ground and excited molecular states, respectively, and the black dot symbolizes a single matter-photon interaction.

where $\mathbf{e}^{(\lambda)}(\mathbf{k})$ is the polarization unit vector $\left[\overline{\mathbf{e}}^{(\lambda)}(\mathbf{k})\right.$ being its complex conjugate], $V$ is an arbitrary quantization volume, and $a^{(\lambda)}(\mathbf{k}), a^{\dagger(\lambda)}(\mathbf{k})$ are, respectively, the photon annihilation and creation operators for a mode $(\mathbf{k}, \lambda)$. The latter operators act on the radiation states through the relations: $a^{(\lambda)}(\mathbf{k})|m(\mathbf{k}, \lambda)\rangle=\sqrt{m}|(m-1)(\mathbf{k}, \lambda)\rangle$, and $a^{\dagger(\lambda)}(\mathbf{k})|m(\mathbf{k}, \lambda)\rangle=\sqrt{m+1}|m+1(\mathbf{k}, \lambda)\rangle$; the appearance of these operators in $H_{\text {int }}$ signifies a photon creation or annihilation.

To secure a general result for the quantum amplitude, $M_{f i}$, of a system, a time-dependent perturbation approach is often applied, which is expressible by

$$
\begin{aligned}
M_{f i}= & \left\langle f\left|H_{\mathrm{int}}\right| i\right\rangle+\sum_{r} \frac{\left\langle f\left|H_{\mathrm{int}}\right| r\right\rangle\left\langle r\left|H_{\mathrm{int}}\right| i\right\rangle}{\left(E_{i}-E_{r}\right)} \\
& +\sum_{s, r} \frac{\left\langle f\left|H_{\mathrm{int}}\right| s\right\rangle\left\langle s\left|H_{\mathrm{int}}\right| r\right\rangle\left\langle r\left|H_{\mathrm{int}}\right| i\right\rangle}{\left(E_{i}-E_{s}\right)\left(E_{i}-E_{r}\right)} \\
& +\sum_{t, s, r} \frac{\left\langle f\left|H_{\mathrm{int}}\right| t\right\rangle\left\langle t\left|H_{\mathrm{int}}\right| s\right\rangle\left\langle s\left|H_{\mathrm{int}}\right| r\right\rangle\left\langle r\left|H_{\mathrm{int}}\right| i\right\rangle}{\left(E_{i}-E_{t}\right)\left(E_{i}-E_{s}\right)\left(E_{i}-E_{r}\right)} \\
& +\cdots, \\
= & M_{f i}^{(1)}+M_{f i}^{(2)}+M_{f i}^{(3)}+M_{f i}^{(4)}+\cdots,
\end{aligned}
$$

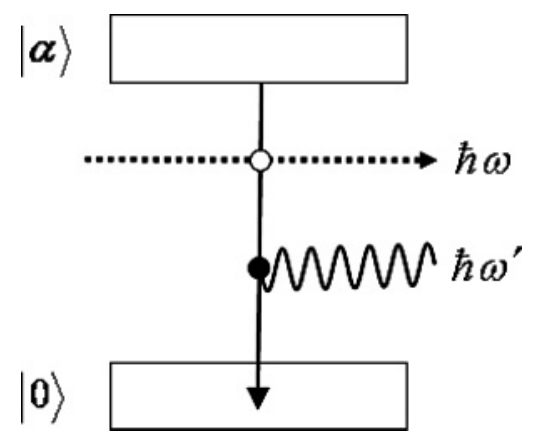

FIG. 2. Energy-level representation for the nonlinear, all-optical control mechanism. The same as in Fig. 1, but also including the off-resonant laser beam $(\hbar \omega)$ denoted by the horizontal dashed arrow; the open dot symbolizes two matter-photon interactions (i.e., elastic forward scattering). where $|i\rangle$ and $|f\rangle$ are, respectively, the initial and final system states (comprising the electronic states of molecule $\xi$ before and after its transition, respectively, and the corresponding radiation states), $|r\rangle,|s\rangle$, and $|t\rangle$ are virtual intermediate states, and $\mathbf{e}_{n}$ is the energy of state $|n\rangle$. Moreover, $M^{(q)}$ represents a quantum amplitude corresponding to $q^{\text {th }}$-order interaction (as denoted by the number of appearances of $H_{\text {int }}$ in the associated term). The rate of a process, $\Gamma$, is determined from Fermi's rule, that is

$$
\Gamma=\frac{2 \pi}{\hbar}\left|M_{f i}\right|^{2} \rho_{f},
$$

where $\rho_{f}$ is the density of radiation states. These QED expressions are the basis for the derivation of a general equation corresponding to the all-optical control mechanism.

\section{GENERAL EXPRESSION}

To elicit details of the theory underlying the phenomenon, it is appropriate to begin by deriving an expression for the observable radiant intensity of fluorescence, correct to third order (i.e., incorporating terms from both first- and third-order perturbation theory). The latter represents the first source of nonlinear correction; even orders vanish since they do not produce final states that differ in only one quantum of radiation from the initial state. The emitted fluorescence power per unit solid angle, $I^{\prime}\left(\Omega^{\prime}\right)$, follows from the Fermi rule rate of Eq. (6) multiplied by the emitted photon energy, $\hbar c k^{\prime}[14,16]$; the result is given by $I^{\prime}\left(\Omega^{\prime}\right) \mathrm{d} \Omega^{\prime}=2 \pi c k^{\prime} \rho_{f}\left|M_{f i}^{(1)}+M_{f i}^{(3)}\right|^{2}$, where $M_{f i}^{(1)}$ and $M_{f i}^{(3)}$ are the quantum amplitudes for the first- and third-order interaction processes, respectively, and $\rho_{f}=\left(k^{\prime 2} V / 8 \pi^{3} \hbar c\right) \mathrm{d} \Omega^{\prime}[8]$. The effects depend on the relative signs of the first- and third-order amplitudes; a common sign will produce fluorescence enhancement, opposite signs its suppression. It is worth passing comment that there is no quantum amplitude corresponding to a linear response to the off-resonant beam, since the development of such an induced moment would engage the electric field of the radiation; as observed in Sec. II, it is apparent from the quantum electrodynamical formulation that this would necessarily create or annihilate a photon. Such a contribution would therefore violate energy conservation within the overall process; this is the same argument that precludes even-order interactions as described above.

The explicit form of the relevant quantum amplitudes are determined by the insertion of Eqs. (2) and (3) into Eq. (5). Spontaneous fluorescence emission is a first-order interaction and hence corresponds to $M_{f i}^{(1)}$. The only states of significance for this process are $|i\rangle=\left|A^{\alpha} ; 0\left(\mathbf{k}^{\prime}\right)\right\rangle$ and $|f\rangle=\left|A^{0} ; 1\left(\mathbf{k}^{\prime}\right)\right\rangle$, where the superscript 0 and $\alpha$ denote the ground and excited states of molecule $A$, respectively. These two system states are of identical energy and, thus, satisfy conservation of energy arguments. Assuming that molecule $A$ is positioned at the origin, the expression $M_{f i}^{(1)}=\mathrm{i}\left(\hbar c k^{\prime} / 2 \varepsilon_{0} V\right)^{\frac{1}{2}} \overline{e_{i}^{\prime}} \mu_{i}^{0 \alpha}$ is determined from the first term of Eq. (5); here, $\mathbf{e}^{\prime}$ is the polarization vector of the fluorescence electromagnetic field, and both the shorthand notation $\boldsymbol{\mu}^{0 \alpha}=\langle 0|\boldsymbol{\mu}| \alpha\rangle$ and the convention of implied index summation are deployed. The nonlinear coupling mechanism is a third-order interaction 


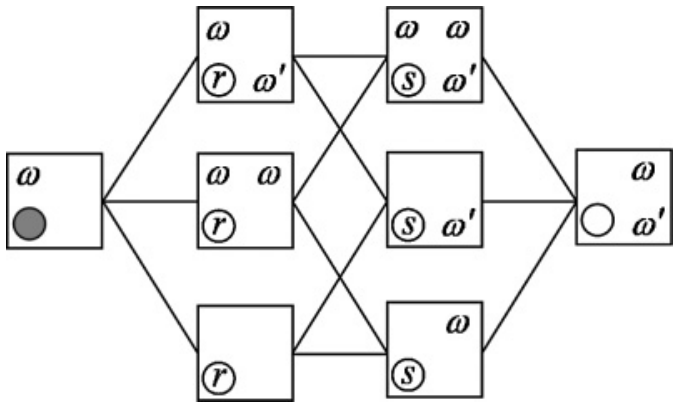

FIG. 3. State-sequence diagram for the all-optical control mechanism. A photon from mode $\omega$ is annihilated and photon modes $\omega^{\prime}$ and $\omega$ are created. The initial state is on the left-hand side, and the final state on the right; each route between the two signifies a different quantum pathway. The solid circle represents the molecule in an excited state, lettered circles an intermediate state, and the open circle a ground state.

and corresponds to $M_{f i}^{(3)}$, which is developed from a sum of six distinct perturbation contributions (i.e., the usual procedure for nonlinear optical systems [16]), rather than one such contribution for $M_{f i}^{(1)}$. To aid the derivation of $M_{f i}^{(3)}$, a state-sequence diagram is constructed (Fig. 3); here, the six perturbation contributions are identified by the six different pathways from the initial to the final state. Details on the procedure to construct such a diagram are to be found in Ref. [17]. From the third term of Eq. (5), with employment of all the possible states and their associated energies (Table I), the following is determined:

$$
M_{f i}^{(3)}=\mathrm{i} m k k^{\prime \frac{1}{2}}\left(\frac{\hbar c}{2 \varepsilon_{0} V}\right)^{\frac{3}{2}} \overline{e_{i}^{\prime}} \bar{e}_{j} e_{k} \beta_{i j k}^{0 \alpha}(\omega)
$$

where $\hbar c k \equiv \hbar \omega$ and $\mathbf{e}$, respectively, denote the energy of a photon and polarization vector of the throughput beam, and $\beta_{i j k}^{0 \alpha}(\omega)$ is to be defined later in the text.

By utilizing the previously derived expressions, correct to third order, the result for the fluorescence intensity contributed

TABLE I. All states and their associated energies for the state sequences exhibited in Fig. 3. Here, $\left|r_{l}^{h}\right\rangle$ represents the system state for which the subscript $l$ is the step number, starting at 0 on the left-hand side, and for each step the superscript $h$ is a vertex index, numbered in sequence from the top.

\begin{tabular}{lcc}
\hline \hline System state $\left|r_{l}^{h}\right\rangle$ & $\left|A^{r} ; \mathbf{k}, \mathbf{k}^{\prime}\right\rangle$ & Energy $E_{r}$ \\
\hline$|i\rangle=\left|r_{0}^{1}\right\rangle$ & $\left|A^{\alpha} ; 1,0\right\rangle$ & $E_{\alpha}+\hbar \omega$ \\
$\left|r_{1}^{1}\right\rangle$ & $\left|A^{r} ; 1,1\right\rangle$ & $E_{r}+\hbar \omega+\hbar \omega^{\prime}$ \\
$\left|r_{1}^{2}\right\rangle$ & $\left|A^{r} ; 0,0\right\rangle$ & $E_{s}+2 \hbar \omega$ \\
$\left|r_{1}^{3}\right\rangle$ & $\left|A^{r} ; 2,0\right\rangle$ & $E_{r}$ \\
$\left|r_{2}^{1}\right\rangle$ & $\left|A^{s} ; 2,1\right\rangle$ & $E_{s}+2 \hbar \omega+\hbar \omega^{\prime}$ \\
$\left|r_{2}^{2}\right\rangle$ & $\left|A^{s} ; 0,1\right\rangle$ & $E_{s}+\hbar \omega^{\prime}$ \\
$\left|r_{2}^{3}\right\rangle$ & $\left|A^{s} ; 1,0\right\rangle$ & $E_{s}+\hbar \omega$ \\
$|f\rangle=\left|r_{3}^{1}\right\rangle$ & $\left|A^{0} ; 1,1\right\rangle$ & $E_{0}+\hbar \omega+\hbar \omega^{\prime}$ \\
\hline \hline
\end{tabular}

by each molecule in the irradiated volume, is given by

$$
\begin{aligned}
I^{\prime}\left(\Omega^{\prime}\right)= & \left(\frac{c k^{\prime 4}}{8 \pi^{2} \varepsilon_{0}}\right) \bar{e}_{i}^{\prime} e_{j}^{\prime}\left[\mu_{i}^{(0) 0 \alpha} \bar{\mu}_{j}^{(0) 0 \alpha}\right. \\
& +\left(I / c \varepsilon_{0}\right) \mu_{i}^{(0) 0 \alpha} \bar{\mu}_{j}^{(2) 0 \alpha}(\omega) \\
& \left.+\left(I^{2} / 4 c^{2} \varepsilon_{0}^{2}\right) \mu_{i}^{(2) 0 \alpha}(\omega) \bar{\mu}_{j}^{(2) 0 \alpha}(\omega)\right],
\end{aligned}
$$

where $I=m \hbar c^{2} k / V$ is the throughput laser irradiance (power per unit area) and, physically, the overbars allow for the possibility of circular polarization. Equation (8) may be interpreted as the emission associated with an effective fluorescence-decay transition moment, and comprises contributions that may be designated $\boldsymbol{\mu}^{(0) 0 \alpha}$ and $\boldsymbol{\mu}^{(2) 0 \alpha}(\omega)$-the former representing the spontaneous (unperturbed) result and the latter a nonlinear correction term that is second order in coupling with the off-resonant radiation of frequency $\omega$ (corresponding to elastic forward scattering). The off-resonant beam thus effects a modification of the decay dipole moment through $\boldsymbol{\mu}^{(2) 0 \alpha}(\omega)$, whose explicit form emerges as

$$
\begin{aligned}
& \mu_{i}^{(2) 0 \alpha}(\omega)=\bar{e}_{j} e_{k} \beta_{i j k}^{0 \alpha}(\omega) \\
& =\bar{e}_{j} e_{k}\left[\sum_{r} \sum_{s \neq \alpha}\left(\frac{\mu_{i}^{0 s} \mu_{j}^{s r} \mu_{k}^{r \alpha}}{\tilde{E}_{s \alpha}\left(\tilde{E}_{r \alpha}-\hbar \omega\right)}+\frac{\mu_{i}^{0 s} \mu_{k}^{s r} \mu_{j}^{r \alpha}}{\tilde{E}_{s \alpha}\left(\tilde{E}_{r \alpha}+\hbar \omega\right)}\right)\right. \\
& \quad+\sum_{r} \sum_{s}\left(\frac{\mu_{j}^{0 s} \mu_{i}^{s r} \mu_{k}^{r \alpha}}{\left(\tilde{E}_{s 0}-\hbar \omega\right)\left(\tilde{E}_{r \alpha}-\hbar \omega\right)}\right. \\
& \left.+\frac{\mu_{k}^{0 s} \mu_{i}^{s r} \mu_{j}^{r \alpha}}{\left(\tilde{E}_{s 0}+\hbar \omega\right)\left(\tilde{E}_{r \alpha}+\hbar \omega\right)}\right) \\
& \left.\quad+\sum_{r \neq 0} \sum_{s}\left(\frac{\mu_{j}^{0 s} \mu_{k}^{s r} \mu_{i}^{r \alpha}}{\left(\tilde{E}_{s 0}-\hbar \omega\right) \tilde{E}_{r 0}}+\frac{\mu_{k}^{0 s} \mu_{j}^{s r} \mu_{i}^{r \alpha}}{\left(\tilde{E}_{s 0}+\hbar \omega\right) \tilde{E}_{r 0}}\right)\right] .
\end{aligned}
$$

Here, $E_{x y}=E_{x}-E_{y}$ is an energy difference between two such states (e.g., $E_{\alpha 0} \equiv \hbar \omega^{\prime}$ ), the transition moments are defined in the same manner as $\boldsymbol{\mu}^{0 \alpha}$, and the excluded summands are due to the rules of perturbation theory. Moreover, the tildes serve as a reminder to add to the excited state energies, in the case of near-resonance conditions, imaginary terms to accommodate damping; explicitly $\tilde{E}_{x y}=E_{x y}-$ $\frac{1}{2} \mathrm{i} \hbar \gamma$, where $\gamma$ represents the full width at half-maximum linewidth near resonance [16]. Also represented in Eq. (9) is the transition hyperpolarizability tensor of the coupling mechanism $\beta_{i j k}^{0 \alpha}(\omega)$. Not to be confused with the state-diagonal hyperpolarizability involved in the Kerr effect, this is a tensor that quantifies the nonlinear engagement of the molecule with the throughput radiation in the course of its decay transition.

The initial term on the right-hand side in Eq. (8) corresponds to spontaneous emission, independent of the probe beam. The third term signifies a coupling of the elastically forward-scattered probe beam with the fluorescence emission. However, it is the second term (linear in I), signifying a quantum interference of these two amplitudes, that represents the leading correction, the focus of our analysis in the following sections. 


\section{THREE-LEVEL SYSTEMS}

For calculational ease, let us assume that the off-resonant probe beam is linearly polarized and all relevant dipole and polarization vectors are broadly parallel. It is then the structure of the energy denominators in Eq. (9) that primarily dictate the degree of enhancement or suppression of the emission. These factors are ultimately determined by the relative positioning of the molecular energy levels, relative to the magnitude of the probe photon energy. To discover more, we can now suppose that the system is three-level-namely $|0\rangle,|\alpha\rangle$, and $|\sigma\rangle$ are the optically prominent states-and focus on the common case where the initially activated level $|\alpha\rangle$ is the lowest electronic excited state (Fig. 4), assuming the same spin multiplicity as the ground state. The probe light is delivered with a tunable beam of frequency $\hbar \omega<E_{\alpha 0}$, precluding excitation from the ground state to higher electronic levels. Maximum effect is achieved if the fluorescent molecule has the electronic state $|\sigma\rangle$ at an energy approximately $\hbar \omega$ above the initial excited state $|\alpha\rangle$, so that $\Delta \tilde{E}=\tilde{E}_{\sigma \alpha}-\hbar \omega$. Effecting the sums over intermediate states $r$ and $s$ in Eq. (9), where each of these states can now be identified with $0, \alpha$ or $\sigma$ (except where excluded by the restrictions on summation), it is the third term on the right that yields the smallest magnitude denominator. We then have

$$
e_{i}^{\prime} \mu_{i}^{(2) 0 \alpha}(\omega) \approx-\frac{\mu^{3}}{\hbar \omega \Delta \tilde{E}}
$$

where it is assumed that $s=0$ and the relevant transition dipoles have broadly equivalent magnitudes (signified by $\mu$ ); such electronic dipole moments are, as usual, assumed to be independent of rovibrational levels. On analysis of Eq. (10), for $E_{\sigma \alpha}<\hbar \omega$ the sign of the leading correction will be positive, signifying that the probe beam enhances fluorescence. In contrast, the sign is negative for $E_{\sigma \alpha}>\hbar \omega$ representing suppressed fluorescence. Typical values of $I^{\prime}\left(\Omega^{\prime}\right)$ may be

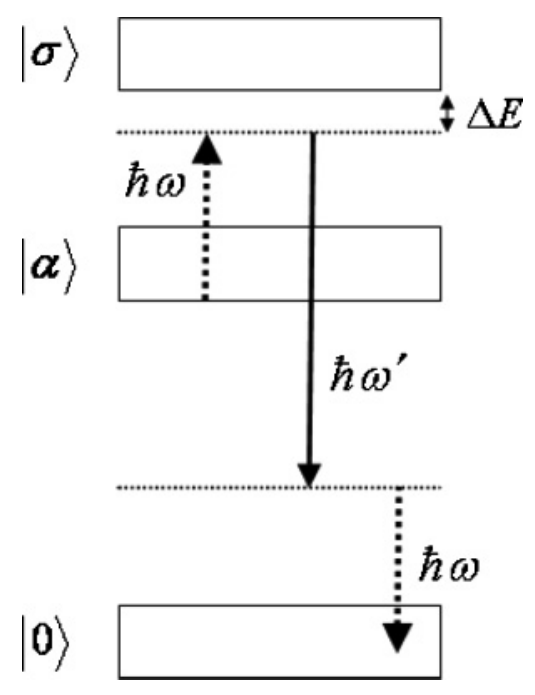

FIG. 4. Energy-level scheme for the nonlinear mechanism based on a three-level system. The same as in Fig. 2, but also including dotted horizontal lines that represent virtual states and $|\sigma\rangle$ as a higher electronic state, which is approximately $\hbar \omega$ above $|\alpha\rangle$ (the difference between which is given by $\Delta E$ ). Dashed vertical arrows are transitions due to the off-resonant laser beam. calculated for various probe laser intensities; setting $\mu=16 \times$ $10^{-30} \mathrm{C} \mathrm{m}, \Delta E=-10^{-20} \mathrm{~J}$, and $\hbar \omega=10^{-19} \mathrm{~J}$, fluorescence is enhanced by $\sim 20 \%$ for an irradiance of $2 \times 10^{15} \mathrm{~W} \mathrm{~m}^{-2}$, and by $\sim 66 \%$ for $I=6 \times 10^{15} \mathrm{~W} \mathrm{~m}^{-2}$. Even at a level of $10^{14} \mathrm{~W} \mathrm{~m}^{-2}$, it can be anticipated that the resulting change in fluorescence intensity will be readily measurable by the use of modulation detection techniques.

\section{CIRCULARLY POLARIZED INPUT}

Let us now suppose the irradiating beam is taken to be circularly polarized. As a result, there is a possibility that the transition dipoles for the nonlinear coupled emission differ for laser input of left $(L)$ and right $(R)$ polarized light. This is quantified by the following expression, which is again determined from the third term of Eq. (9):

$$
\begin{aligned}
\Delta \mu_{i}^{(2) 0 \alpha}(\omega) & =\mu_{i}^{(2) 0 \alpha}\left(\omega_{L}\right)-\mu_{i}^{(2) 0 \alpha}\left(\omega_{R}\right) \\
& \approx \frac{i \mu_{i}^{0 \sigma} \mu_{j}^{00} \mu_{k}^{\sigma \alpha} \hat{k}_{l} \varepsilon_{j k l}}{\hbar \omega \Delta \tilde{E}}
\end{aligned}
$$

where $e_{j}^{(L)} \bar{e}_{k}^{(L)}-e_{j}^{(R)} \bar{e}_{k}^{(R)}=-\mathrm{i} \hat{k}_{l} \varepsilon_{j k l}$ [13], in which $\hat{\mathbf{k}}$ is the wave vector of the throughput laser beam. For a nonvanishing result it is necessary that $\mu_{j}^{00}, \mu_{k}^{\sigma \alpha}$, and $\hat{k}_{l}$ are noncoplanar; assuming for simplicity that these vectors are mutually orthogonal, then by inserting Eq. (11) into the leading correction term of Eq. (8) the difference in magnitude of the fluorescence intensity for left and right polarized beams is determined, and the result is given by

$$
\Delta I^{\prime}\left(\Omega^{\prime}\right)=\left(\frac{I k^{\prime 4}}{16 \pi^{2} \varepsilon_{0}^{2} \omega}\right) \frac{\mu^{4} \gamma}{\left(\Delta E^{2}+\frac{1}{4} \hbar^{2} \gamma^{2}\right)},
$$

where damping is now explicit. It is notable that the result signifies a form of chiral differentiation associated with the imaginary part of the transition moment, whose origin lies in the complex transition hyperpolarizability-the term within square brackets in the right-hand side of Eq. (9). This form of behavior tallies exactly with the case of surface second harmonic generation, where readily observable chiral discrimination also arises from imaginary contributions to a complex molecular hyperpolarizability [18-21]. In both cases, it is the proximity to resonance that inflates the imaginary component, leading to an observable dependence on handedness. In the present case, employing typical values for $\mu, \Delta E$, and $\hbar \omega$ (as given earlier), and $\Delta E \approx \hbar \gamma$, the intensity difference of $\Delta I^{\prime}\left(\Omega^{\prime}\right)$, expressed as a ratio relative to the spontaneous fluorescence intensity $I^{\prime}\left(\Omega^{\prime}\right)$, is $\sim 8 \%$ for an irradiance of $2 \times 10^{15} \mathrm{~W} \mathrm{~m}^{-2}$, and $\sim 23 \%$ for $I=6 \times 10^{15} \mathrm{~W} \mathrm{~m}^{-2}$. Again, significantly lower levels of intensity should prove feasible using modulation detection.

\section{CONCLUSION}

The typical figures presented above prove that significant modification of fluorescence should be experimentally obtainable. We have also shown that, on approaching resonance conditions, the use of a circular polarized probe beam delivers additional, chirally sensitive features; the mechanism does not require the presence of a surface. It can be envisaged that time-correlated photon techniques might represent a 
methodology particularly well suited to the detection of change in the fluorescence intensity, deployed in an adaptation of the instrumentation for standard laser fluorescence spectroscopy. The inclusion of quarter-wave plates would facilitate resolution of the circular polarization features that we have identified. With a tunable laser source, the capacity to control the wavelength of the throughput radiation introduces an additional experimental variable, offering a multiple field dimension to sample interrogation; establishing the variation with laser pulse intensity will also validate the mechanism at work.

Having established the technical viability of experimental detection of the all-optical control process, it is worth considering whether any competing processes could interfere with its measurement. Since the throughput radiation is designedly off-resonant, such processes could not involve absorption or stimulated emission, but light scattering does invite attention. Specifically, it might be supposed that, at the levels of intensity our experiment requires, a process of electronic anti-Stokes Raman scattering could also serve to deliver the molecular decay transition. However, the possible difficulties that such a process might introduce are easily addressed by some of the same methods that are routinely used to discriminate Raman from fluorescence signals, particularly with regard to detection of the optical emission-which in this case would not be seen at the fluorescence wavelength, but at a much shorter wavelength, its optical frequency corresponding to a sum of the fluorescence and the laser frequencies. It is also to be observed that the selection rules for the two types of process are different, since electronic Raman scattering is associated with two-photon selection rules, whereas the process described in this article satisfies one- and three-photon rules.

Although the present results are valid for systems in which the fluorophores are orientationally ordered, future work will address cases where there is disorder or rotational freedom, including calculations of the associated fluorescence anisotropy. Beyond these considerations, we can envisage substantial scope for further investigations focusing on specific molecular systems, through explicit calculation of the electronic state energies and transition dipole elements, thereby facilitating a precise interpretation of the corresponding experimental results. In summary, our quantum electrodynamical calculations have demonstrated the achievability of all-optical control of molecular fluorescence, operated through the input of off-resonant laser light. We propose that this novel all-optical control mechanism introduces new perspectives to molecular fluorescence spectroscopy, microscopy imaging, and circular dichroism.

\section{ACKNOWLEDGMENTS}

The authors are grateful to an anonymous referee for insightful comments, and to the Leverhulme Trust for financial support.
[1] H. Rigneault, J. Capoulade, J. Dintinger, J. Wenger, N. Bonod, E. Popov, T. W. Ebbesen, and P.-F. Lenne, Phys. Rev. Lett. 95, 117401 (2005).

[2] P. Anger, P. Bharadwaj, and L. Novotny, Phys. Rev. Lett. 96, 113002 (2006).

[3] F. D. Stefani, K. Vasilev, N. Bocchio, N. Stoyanova, and M. Kreiter, Phys. Rev. Lett. 94, 023005 (2005).

[4] S. Kühn, U. Håkanson, L. Rogobete, and V. Sandoghdar, Phys. Rev. Lett. 97, 017402 (2006).

[5] M. Ringler, A. Schwemer, M. Wunderlich, A. Nichtl, K. Kürzinger, T. A. Klar, and J. Feldmann, Phys. Rev. Lett. 100, 203002 (2008).

[6] G. Vecchi, V. Giannini, and J. Gómez Rivas, Phys. Rev. Lett. 102, 146807 (2009).

[7] A. Chizhik, F. Schleifenbaum, R. Gutbrod, A. Chizhik, D. Khoptyar, A. J. Meixner, and J. Enderlein, Phys. Rev. Lett. 102, 073002 (2009)

[8] L. Mandel and E. Wolf, Optical Coherence and Quantum Optics (Cambridge University Press, Cambridge, 1995).

[9] L. Kastrup, H. Blom, C. Eggeling, and S. W. Hell, Phys. Rev. Lett. 94, 178104 (2005).
[10] S. W. Hell, Science 316, 1153 (2007).

[11] P. Allcock, R. D. Jenkins, and D. L. Andrews, Phys. Rev. A 61, 023812 (2000).

[12] D. L. Andrews, Phys. Rev. Lett. 99, 023601 (2007).

[13] D. S. Bradshaw and D. L. Andrews, J. Chem. Phys. 128, 144506 (2008).

[14] D. P. Craig and T. Thirunamachandran, Molecular Quantum Electrodynamics (Dover, Mineola, New York, 1998).

[15] R. G. Woolley, Adv. Quantum Chem. 32, 167 (1999).

[16] D. L. Andrews and P. Allcock, Optical Harmonics in Molecular Systems (Wiley VCH, Weinheim, 2002).

[17] D. L. Andrews and D. S. Bradshaw, Eur. J. Phys. 30, 239 (2009).

[18] J. D. Byers, H. I. Yee, and J. M. Hicks, J. Chem. Phys. 101, 6233 (1994).

[19] J. M. Hicks, T. Petralli-Mallow, and J. D. Byers, Faraday Discuss. 99, 341 (1994).

[20] G. J. Simpson, J. Chem. Phys. 117, 3398 (2002).

[21] G. J. Simpson, ChemPhysChem 5, 1301 (2004). 